\title{
- Development and Testing of a Multimodal Acquisition Platform for Human-Robot Interaction Affective Studies
}

\author{
Nicole Lazzeri, Daniele Mazzei, and Danilo De Rossi \\ Research Center "E. Piaggio," University of Pisa, Italy
}

Human-Robot Interaction (HRI) studies have recently received increasing attention in various fields, from academic communities to engineering firms and the media. Many researchers have been focusing on the development of tools to evaluate the performance of robotic systems and studying how to extend the range of robot interaction modalities and contexts. Because people are emotionally engaged when interacting with computers and robots, researchers have been focusing attention on the study of affective human-robot interaction. This new field of study requires the integration of various approaches typical of different research backgrounds, such as psychology and engineering, to gain more insight into the human-robot affective interaction.

In this paper, we report the development of a multimodal acquisition platform called HIPOP (Human Interaction Pervasive Observation Platform). HIPOP is a modular data-gathering platform based on various hardware and software units that can be easily used to create a custom acquisition setup for HRI studies. The platform uses modules for physiological signals, eye gaze, video and audio acquisition to perform an integrated affective and behavioral analysis. It is also possible to include new hardware devices into the platform. The open-source hardware and software revolution has made many high-quality commercial and open-source products freely available for HRI and HCI research. These devices are currently most often used for data acquisition and robot control, and they can be easily included in HIPOP.

Technical tests demonstrated the ability of HIPOP to reliably acquire a large set of data in terms of failure management and data synchronization. The platform was able to automatically recover from errors and faults without affecting the entire system, and the misalignment observed in the acquired data was not significant and did not affect the multimodal analysis. HIPOP was also tested in the context of the FACET (FACE Therapy) project, in which a humanoid robot called FACE (Facial Automaton for Conveying Emotions) was used to convey affective stimuli to children with autism. In the FACET project, psychologists without technical skills were able to use HIPOP to collect the data needed for their experiments without dealing with hardware issues, data integration challenges, or synchronization problems. The FACET case study highlighted the real core feature of the HIPOP platform (i.e., multimodal data integration and fusion). This analytical approach allowed psychologists to study both behavioral and psychophysiological reactions to obtain a more complete view of the subjects' state during interaction with the robot.

These results indicate that HIPOP could become an innovative tool for HRI affective studies aimed at inferring a more detailed view of a subject's feelings and behavior during interaction with affective and empathic robots.

Keywords: human-robot interaction, affective computing, multimodal approach, physiological signals

\footnotetext{
Authors retain copyright and grant the Journal of Human-Robot Interaction right of first publication with the work simultaneously licensed under a Creative Commons Attribution License that allows others to share the work with an acknowledgement of the work's authorship and initial publication in this journal.
}

Journal of Human-Robot Interaction, Vol. 3, No. 2, 2014, Pages 1-24, DOI 10.5898/JHRI.3.2.Lazzeri 


\section{Introduction}

Human-Robot Interaction (HRI) is a multidisciplinary field that draws from both Human-Machine and Human-Computer Interaction (HMI/HCI) and attracts researchers from various areas, such as artificial intelligence, robotics, psychology, cognitive sciences, and affective computing.

Unlike early autonomous robots that performed tasks requiring minimal interaction with humans, social robots are specifically designed to interact with people and establish a social relationship. In particular, humanoid social robots interact with people through natural communication channels, such as facial expressions, body gestures, and speech to meet the expectations of their human partners (Bethel, Burke, Murphy, \& Salomon, 2007; Robinson \& El Kaliouby, 2009).

As robots become increasingly social and interactive, the study of HRI needs to address a wide variety of challenges. One of these concerns the development of methods for understanding and evaluating how people interpret human-like signs expressed by social robots and how people feel when interacting with these synthetic partners.

Human-robot interaction studies are usually based on the acquisition and analysis of three types of data (Bethel et al., 2007; Kidd \& Breazeal, 2005): self-report annotations, behavioral observations, and psychophysiological measures.

Self-report annotations concern study participants' notes about their mood and feelings during interaction with robots. This information can be gathered by researchers by posing questions to participants during or after the interaction (Bradley \& Lang, 1994). Participants may answer in a manner that depends on their emotional state or they may be unable to explain exactly how they feel. For instance, people could lie about their mood or feelings for emotionally perceived reasons, such as humiliation or embarrassment associated with their inability to accomplish the requested task.

Behavioral observations are based on the interpretation of data gathered from participants' actions during interaction with robots. These data are acquired using multiple cameras, microphones, and software tools that log the participants' activities (Bethel \& Murphy, 2010). The analysis of the participants' behavior and reactions provides fundamental information for overall interpretation, but the results can often be misleading. For instance, two participants could feel different emotions but behave in the same way, or conversely, they could have the same feelings while displaying completely different reactions.

Psychophysiological measures offer non-invasive methods for monitoring physiological correlates of subjects' emotional states. These measures are more objective than self-report annotations and behavioral observations, because they depend on autonomic nervous system (ANS) activity. For this reason, physiological parameters such as heart rate variability (HRV) and electrodermal response (EDR) are widely used in affective computing and in HRI/HCI studies (Andreassi, 2000). However, the acquisition and analysis of physiological signals pose different challenges related to the quality of the recording, which requires synchronicity, noise cancellation, and motion artifact reduction in addition to the management of specific sensors and the setup of an appropriate laboratory (Tiberio, Cesta, \& Olivetti Belardinelli, 2013). Physiological signals are also affected by the so-called "law of initial values," which holds that the response to a stimulus is dependent on the initial baseline level (Stern, Ray, \& Quigley, 2001); therefore, dedicated planning of the experiment is fundamental for the acquisition of usable and reliable data. However, data of this type do not offer conclusive and unique evidence of subjects' emotional state and have to be considered as a piece of information about the overall subjects' state (Cacioppo, 2007).

Each type of investigation previously described has its advantages and disadvantages, and none of them is sufficient on its own to completely evaluate and understand participants' reactions to interactions with robots (Kidd \& Breazeal, 2005; Steinfeld et al., 2006). Indeed, the comparison and combination of data obtained using different methods provide a way to extract additional information about the subject's state by the interaction. This information comes from the 
interpretation of the entire "puzzle" in which apparently misaligned or conflicting evidence can hide other important information.

Ultimately, multimodal data acquisition and processing are difficult tasks for HRI experimenters because they require complex technical equipment composed of different hardware and software tools. For instance, hardware devices used for physiological data collection, video acquisition, annotation software and robot control frameworks are usually based on proprietary software with dedicated communication protocols. This non-homogeneous condition makes the data gathering and processing a very difficult task that is beyond the focus and expertise of most HRI researchers.

In this paper, we report the development of an innovative platform for HRI multimodal studies called HIPOP (Human Interaction Pervasive Observation Platform). HIPOP is a modular data acquisition platform designed for the setup of multimodal data acquisition experiments on which humans' reactions during interactions with robots are investigated with a specific focus on affective behaviors. HIPOP is designed to be a flexible and reconfigurable platform on which all the methods described above are integrated in overcoming the typical problems associated with protocols, systems integration, data synchronicity and acceptability of acquisition devices.

Thanks to the open-source hardware and software revolution, various high-quality products used in HRI and HCI research are freely available (some examples reported in Table 1). HIPOP is designed with a special focus on the "Open Source" philosophy, allowing experimenters to easily integrate dedicated acquisition modules based on commercial products' API (application programming interface) or on open-source hardware and software solutions. HIPOP includes a dedicated software module for data acquisition through serial ports to integrate "open" devices based on Arduino, RaspBerryPi, and other similar products. These single-board devices are widely used for acquiring data and controlling robots and other devices while allowing experimenters to integrate a wide range of stimuli conveying and data acquisition systems in HIPOP.

\section{Related Works}

Multimodal acquisition and analysis methods have been proposed in many different types of experiments to demonstrate how it is possible to draw a more detailed picture of the HRI and HMI outcomes through the integration of different types of data and observation modalities (Jaimes \& Sebe, 2007). Various mathematical and statistical methods for multimodal data fusion have also been developed aimed at extracting common indicators of subjects' states from different types of data, which can be correlated with the robot interaction (Atrey, Hossain, El Saddik, \& Kankanhalli, 2010).

Wienke, Klotz, and Wrede (2012) presented a conceptual framework for acquiring a multimodal data set, taking care of synchronization, automation, and validation of recorded data. Moreover, they highlighted the importance of devices calibration procedure, developing reusable tools and components that maintain the data set calibration.

The synchronization between devices used for data acquisition during human-robot interactions has also been analyzed by Lohse, Hanheide, Rohlfing, and Sagerer (2009), who developed Systemic Interaction Analysis (SInA), a platform for the correlation of performances of robot system modules with subjects' reactions. The system records data in the form of video and $\log$ files, allowing experimenters to perform a qualitative analysis aimed at identifying prototypical interaction sequences that can be correlated with quantitative measures of the robot's state.

Various works have also been carried out regarding the identification of quantitative indexes used as evaluators of HRI interactions. Dautenhahn and Werry (2002) developed a micro-behaviors quantitative analysis technique that scores video recordings for extracting different kinds of indicators. 
Lisetti and Nasoz (2002) developed a framework called Multimodal Affective User Interface (MAUI), which allows a virtual agent to perceive its interlocutor's emotional state through multimodal data fusion. The agent, being conscious of the interlocutor's state, can react with emotionally related actions establishing an empathic relationship. MAUI performs emotion recognition through the integration of automatic facial expression recognition systems, wearable computers for the acquisition of psychophysiological signals, and annotations of the users' dialogs.

Table 1. Examples of open- source hardware, software acquisition units, and robots widely used in current robotics research.

\begin{tabular}{|c|c|c|}
\hline Software Tools & $\begin{array}{l}\text { Description } \\
\end{array}$ & URL \\
\hline OpenVibe & $\begin{array}{l}\text { Software platform dedicated to } \\
\text { designing, testing and using brain- } \\
\text { computer interfaces }\end{array}$ & http://openvibe.inria.fr \\
\hline Kubios & $\begin{array}{l}\text { Heart rate variability analysis } \\
\text { software }\end{array}$ & http://kubios.uef.fi \\
\hline Ledalab & $\begin{array}{l}\text { Software for the analysis of skin } \\
\text { conductance data }\end{array}$ & http://www.ledalab.de \\
\hline Neuro Server & $\begin{array}{l}\text { EEG server for acquisition and } \\
\text { streaming of raw signals }\end{array}$ & http://openeeg.sourceforge.net \\
\hline Arduino & $\begin{array}{l}\text { Open-source hardware electronic } \\
\text { prototyping platform widely used } \\
\text { in robotics research }\end{array}$ & http://www.arduino.cc \\
\hline RaspBerry Pi & $\begin{array}{l}\text { 32-bit single-board computer with } \\
\text { open-source hardware and software }\end{array}$ & http://www.raspberrypi.org \\
\hline E-Health & $\begin{array}{l}\text { Sensor platform for biometric data } \\
\text { acquisition with Arduino and } \\
\text { Raspberry Pi }\end{array}$ & $\begin{array}{l}\text { http://www.cooking- } \\
\text { hacks.com }\end{array}$ \\
\hline Emotiv & $\begin{array}{l}\text { High-resolution, multi-channel, } \\
\text { wireless neuro-headset with open } \\
\text { API }\end{array}$ & http://www.emotiv.com \\
\hline Kinect & $\begin{array}{l}\text { 3D video acquisition systems with } \\
\text { open-source drivers }\end{array}$ & $\begin{array}{c}\text { http://www.microsoft.com/en- } \\
\text { us/kinectforwindows }\end{array}$ \\
\hline Aldebaran NAO & $\begin{array}{l}\text { One-meter tall walking robot } \\
\text { widely used in HRI with extended } \\
\text { APIs }\end{array}$ & $\begin{array}{l}\text { http://www.aldebaran- } \\
\text { robotics.com }\end{array}$ \\
\hline Hanson RoboKind & $\begin{array}{l}\text { One-meter tall walking robot with } \\
\text { open-source control code and APIs }\end{array}$ & http://hansonrobokind.com \\
\hline ICUB & $\begin{array}{l}\text { High performance humanoid robot } \\
\text { with control system based on the } \\
\text { YARP open-source framework }\end{array}$ & http://www.icub.org \\
\hline
\end{tabular}

Most of these scientific works report the development of an acquisition platform specifically tailored for the designed experiment. HIPOP has been designed to overcome this limitation by allowing experimenters to use a modular and widely configurable open-source platform. In HIPOP, each software block is a reusable module that can be run as a standalone program to 
perform single acquisition or simple experiments. The same HIPOP modules can be also used in a more complex multimodal setup.

In today's field of behavioral analysis, various software solutions for video annotations, data gathering, and data analysis are available. According to the Wikipedia page, Comparison of behavioral experiment software1, only 14 out of 25 reported software have a Graphical User Interface (GUI) for a user-friendly interaction. Only five of these GUI-based behavioral analysis software are free and only three of them have extension libraries that allow users to interface with other.

In particular, Noldus Observer 2, one of the most used commercial video annotation tools, integrates a software development kit (SDK) that allows users to run, drive, and trigger other software actions when buttons on the Observer's GUI are pressed or when particular routines are run. These types of extensions are widely used to integrate behavioral data with devices for gaze and physiological signal acquisition.

As open-source framework for parallel acquisition of multiple sensors, HIPOP can also be used as a software connection between behavioral analysis tools that make SDK and acquisition devices available, allowing easy integration of the acquired data.

\section{Research Approach}

The development of HIPOP was motivated by the following statement about emotions: "...emotions are more or less the complex reactions the body has to certain stimuli. When we are afraid of something, our hearts begin to race, our mouths become dry, our skin turns pale, and our muscles contract. This emotional reaction occurs automatically and unconsciously." (Damasio, 2005). Damasio's book, Descartes' Error, illustrates that emotions allow human beings to make decisions, because the process of monitoring the internal state of the body by the brain and the process of generating emotions are closely connected and involve the same brain areas (Damasio, 1994). Consequently, emotions are perceived and expressed as complicated blends of body gestures, actions, involuntary behaviors, and physiological correlates that can be investigated only if observed as a set of emotional outputs on the whole (Ledoux, 1998).

For this reason, the approach of HIPOP is focused on the analysis of the subjects' reactions to the stimuli conveyed by the robot. Therefore, the robot is a fundamental part of the HIPOP acquisition setup. Robot's actions are stored and then analyzed together with subject's data in order to investigate HRI dynamics.

Fig. 1 shows the HIPOP data flow. Different data types are considered in HIPOP as separated inputs with dedicated preprocessing and pre-analysis procedures.

During the preprocessing phase, subjects' answers and experimenters' annotations are scored while video/audio recordings and physiological signals are filtered to remove noise and motion artifacts. Scores of the subjects' answers and therapists' annotations are statistically analyzed, while video and audio files are analyzed to identify time references of the protocol phases. Video and audio files are also analyzed to annotate each subject's gestures and behaviors for inferring the subject's reactions.

Audio signals, together with filtered physiological measures, are used to extract high-level features that can be correlated with the subject's psychophysiological state. Extracted features are statistically processed in order to identify correlations between the protocol phases and the subject's psychophysiological state. In this phase, if the experiment involves statistically different populations, a comparison between them is performed in order to identify how different groups react to stimuli conveyed by the robot.

All analysis phases use the robot log as reference for the identification of stimuli type and time, allowing experimenters to perform a correlation analysis. The high-level multimodal analysis of HIPOP allows experimenters to extract information about subject's reactions to the 
robot (i.e., comfort and expectation in interacting with the robot) and the type of interaction (i.e., how much the robot was able to involve the subject and convey emotional information). Through data fusion, behavioral information analysis allows experimenters to infer the subject's experience in terms of engagement and emphatic attitudes in the human-robot social interaction.

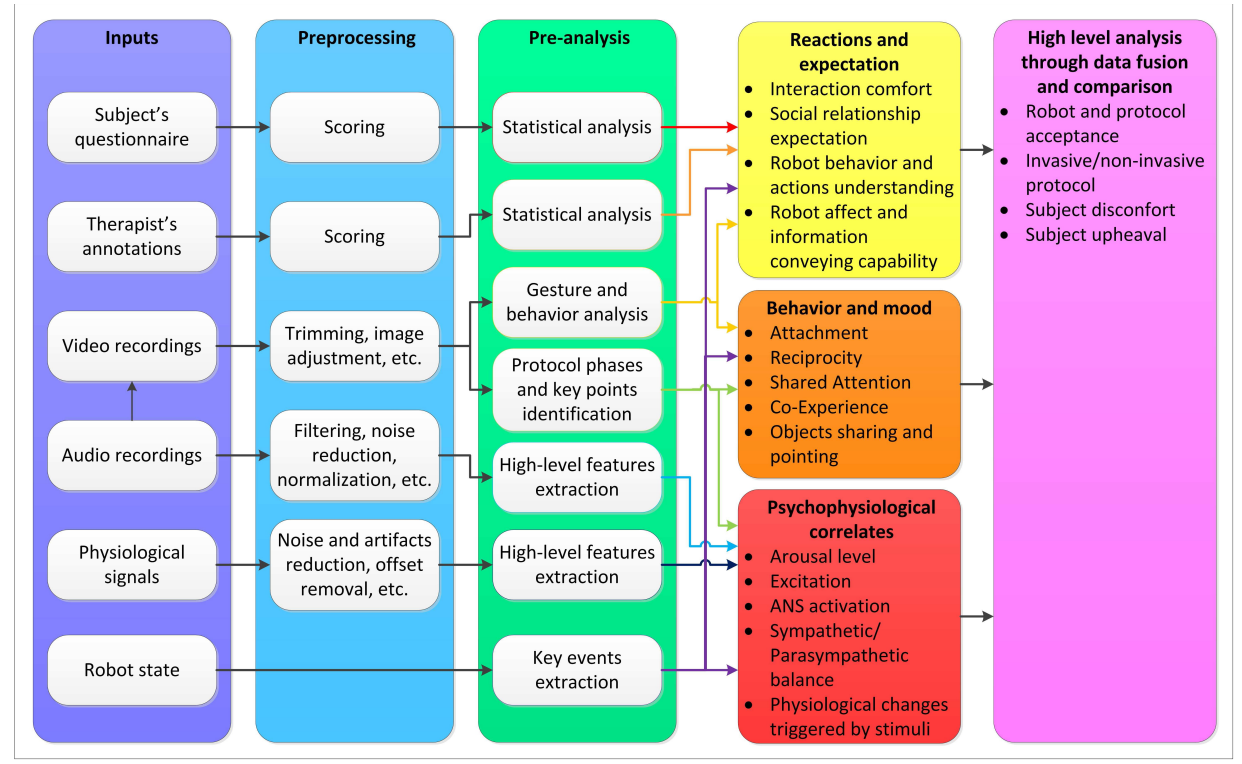

Figure 1. Data flow for the multimodal analysis approach used in HIPOP.

Lastly, all information obtained in the previous analysis steps can be compared and fused together to obtain a deeper understanding and a more detailed interpretation of the subject's interaction with the robot. The data fusion process is not a simple merger of different data types. Data are compared to highlight apparently misaligned signs that could contain crucial information about the subject's interpretation of feelings. For example, a subject could act calm and claim to be confident during a social interaction, while actual physiological parameters could highlight a stressed or altered state in conflict with the subject's claim. These observations could appear to be inconsistent. This apparent affective misalignment requires scientists to ascertain affective content information that is not obvious, which is difficult to do through classical behavioral analysis. The explanation may be that the subject was embarrassed about declaring discomfort during interaction with the robot (J. Kim \& André, 2008; K. Kim, Bang, \& S. Kim, 2004).

A very important aspect in psychophysiological acquisition experiments is related to the need for pervasive, ubiquitous, and unobtrusive data acquisition approaches. Pervasiveness in HRI studies cannot be achieved by simply increasing the number of sensors worn by the subject. Pervasiveness requires comprehensive observation of the subject's behavior without interfering with that behavior. Ubiquity means introducing sensors for data acquisition and computation that are highly interwoven into the physical environment. Unobtrusiveness is crucial for effective and non-invasive data acquisition; uncomfortable garments or physical limitations have been found to introduce bias in the subject's affective state (Stern et al., 2001).

Moreover, we need the flexibility that reusable software blocks can provide, because the range of possible HRI studies is very wide and interaction tasks can span from physical to emotional human-robot interactions. As previously mentioned, HIPOP was specifically developed to address this issue. The platform can be reconfigured easily without requiring software or 
hardware redesign. This feature allows experimenters to decide how many modules of each preconfigured type are needed for the experiment or to easily develop their own additional modules for the integration of new sensors and hardware.

Psychophysiological experiments are usually associated with behavioral analysis performed through ad-hoc annotation tools. Most of the commercial video annotation tools make an SDK available to interface with a particular programming language or to communicate with certain libraries or embedded systems. In these cases, HIPOP can be used as an interconnection system between the annotation tool and the sensors designed for the experiment. Indeed, the experimenter could design and develop a HIPOP module that interfaces with both the acquisition device and the SDK of the video annotation tool. This software connection would allow the experimenter to control the entire HIPOP system directly from the annotation software and easily connect the acquisition devices with the preferred annotation tool.

\section{Materials and Methods}

HIPOP is a multichannel acquisition platform aimed at extracting a large amount of various types of data. The multimodal data fusion approach used in HIPOP makes it possible to infer new affective information, both in real time and off-line, for continuous monitoring of the robot-subject interaction and a thorough evaluation of the experiment.

\subsection{The Underlying Framework}

The HIPOP architecture is based on Robotics4.NET (Cisternino, Colombo, Ennas, \& Picciaia, 2005), a programming framework inspired by a biological model. The main concept is shaped around the notion of the Body, a sensory system that acquires data from the environment; the Brain, which manages and uses the acquired data by triggering body actions if necessary; and the Bodymap, which acts as a shared blackboard for the communication between the Body and the Brain. The Body is composed of various software modules called Roblets that can be considered the sensing apparatus of an organism.

The extreme versatility and adaptability of Robotics4.NET makes it a good choice for the development of modular platforms. In particular, systems based on this framework are able to be self-reconfigurable in case of errors. On one hand, if a Roblet crashes, it can be disconnected and reconnected automatically without compromising the entire system. On the other hand, if the Bodymap crashes, all the Roblets can continue their tasks of collecting, storing, and visualizing data. Once the Bodymap is started again, the exchange of messages between the Bodymap and the active Roblets restarts. The loss of messages is not critical because the Roblets communicate continuously with the Bodymap. If a message gets lost, the information will be delivered with the next message.

\subsection{The HIPOP Architecture}

HIPOP includes a set of hardware modules that can be connected together into a desired configuration. The various hardware devices, especially if they have different manufacturers, have their own proprietary software and communication protocol. A Roblet functions as the interface between the hardware device and the Bodymap. A Roblet allows the user to configure a hardware device and record and visualize the data acquired with that device in real time. These data can be transmitted to the Bodymap to monitor the experiment through the Bodymap viewer (as described in section 4.3.7).

Each Roblet is controlled by the Bodymap, which sends messages to start, stop, and pause synchronization of the data flow. Once the Bodymap is started, it sends a timestamp to all Roblets that is used as a time reference for guaranteeing the synchronicity and alignment of the Roblet's locally stored data. 
HIPOP also includes an annotation system to insert key point references in the stored data. Key point references can be added by experimenters though an "insert key point" button in the Roblet interface or through a dedicated button of the Bodymap viewer, which then sends a broadcast message to all Roblets for triggering a local key point annotation event.

Data acquired by each Roblet is locally stored as XML files. All XML files are manually collected at the end of the experiment and merged in a single data set through a dedicated data management tool (see section 4.3.8).

\subsection{The HIPOP Modules}

Currently, HIPOP includes six acquisition modules and a supervising interface called Bodymap viewer. HIPOP modules can be used all together, as standalone applications for single measurement acquisition, or in subsets according to the experiment requirements and setup.

The following sections describe current details of the HIPOP modules.

\subsubsection{The FACE robot state recording module.}

The FACE is a humanoid developed in collaboration with Hanson Robotics and consists of a passive, articulated body equipped with an artificial skull. The FACE is actuated by 32 servo motors that allow the robot to reproduce various human-like expressions (Fig. 2) and control its eye and neck movements using a dedicated algorithm (Mazzei, Lazzeri, Hanson, \& De Rossi, 2012).

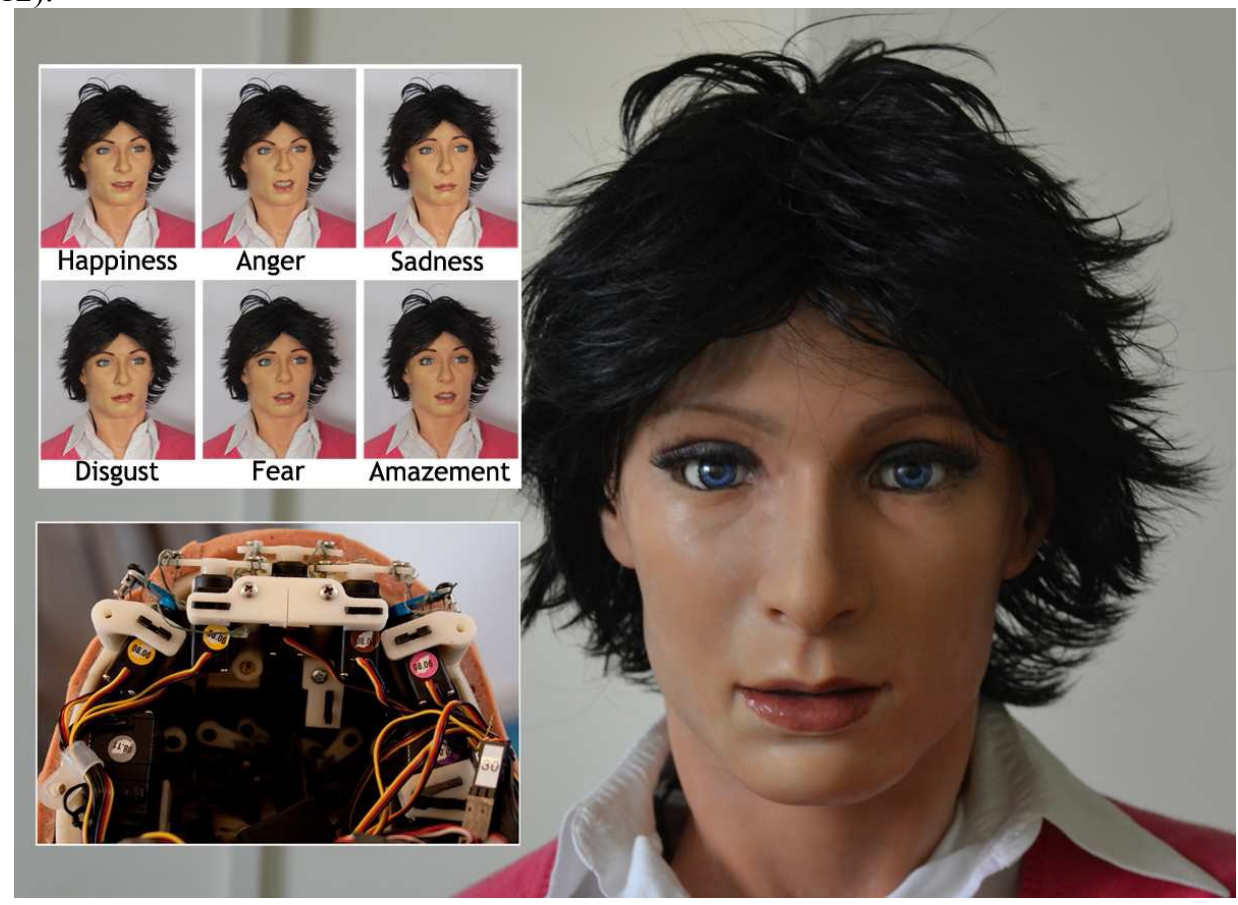

Figure 2. The FACE android.

The android has a CCD camera in the right eye that is used for tracking the subject's face and a high-definition camera in the left eye that is used to record the subject's facial features and expressions. The FACE can also speak through a text-to-speech synthesizer that is manually controlled by the operator, even if lip syncing is not yet integrated into the FACE.

A software module called FACE Roblet was developed for integrating the robot FACE into the platform and storing the robot state during the interaction. The FACE state is logged locally as 
an XML file and transmitted to the Bodymap viewer, allowing experimenters to monitor the robot's actions, behaviors, and parameters in real-time.

The synchronous acquisition of the FACE state with all other data is fundamental in a multimodal data analysis to infer the subject's psychophysiological and behavioral state through the correlation between the robot's actions and the subject's signals.

Data stored by the FACE Roblet include:

- expressions performed by the FACE;

- neck joint coordinates for identifying the robot's head direction;

- eye motor coordinates for identifying the robot's gaze.

Optionally, it is also possible to record video stream with the camera in the FACE's right eye.

\subsubsection{Motorized cameras and microphones acquisition module}

Video recording is fundamental in HRI behavioral analysis (Kanda, Ishiguro, Imai, \& Ono, 2003; Kanda, Miyashita, Osada, Haikawa, \& Ishiguro, 2008). Typical HRI experiments are conducted in rooms equipped with motorized pan/tilt/zoom cameras mounted in opposite corners. Cameras are used to record the subject's behavior and actions from different points of view (i.e., one camera can be focused on the subject's face while the other is focused on the entire room). A wearable microphone is usually used for recording the subject's voice, while an environmental microphone records the environmental sounds.

A software module called Video Roblet was developed to record video stream from cameras and audio stream from microphones. The Video Roblet interfaces with the video and audio acquisition boards allowing experimenters to configure various settings, such as the video and audio resolution, the frame rate, and the audio sample rate. The video stream is recorded locally on the machine that hosts the Video Roblet, but it is also reduced in resolution and transmitted to the Bodymap viewer allowing experimenters to monitor the interaction outcome in real-time.

The Video Roblet previews the video when it is started, which allows experimenters to adjust camera positions and zoom. Video recording starts when the Bodymap sends the "record" message in broadcast synchronously to the other active Roblets. As previously mentioned, the Video Roblet can receive an "insert key point" message to annotate time event references. The Video Roblet can also receive a "take a picture" message to capture snapshots of the scene.

Video and audio recordings are used by the experimenters to evaluate the subject's behavior and body language in order to identify protocol phases and relevant events. Audio recordings obtained with the wearable microphone can also be used to perform audio affective studies and annotate the subject's answers and vocalizations.

\subsubsection{Sensorized t-shirt acquisition module}

The subject's physiological signals are acquired through an unobtrusive sensorized t-shirt developed using the e-textiles technique by Smartex (Paradiso, Loriga, \& Taccini, 2005). The sensorized t-shirt has been proven to deliver high-quality data while being unobtrusive and very comfortable (Scilingo et al., 2005). An embedded acquisition electronic unit equipped with a Bluetooth wireless connection is used to record, preprocess, and transmit physiological signals to a PC using a dedicated Bluetooth dongle (Fig. 3).

The sensorized t-shirt provides real-time acquisition of electrocardiogram (ECG) and respiratory signals. The electronic acquisition unit includes a three-axis accelerometer. The primary signals (i.e., ECG, respiration, and the three-axis accelerometer activity) are acquired and preprocessed by an on-board electronic acquisition unit. Raw and processed data (Table 2) are transmitted to a PC through a Bluetooth connection. 

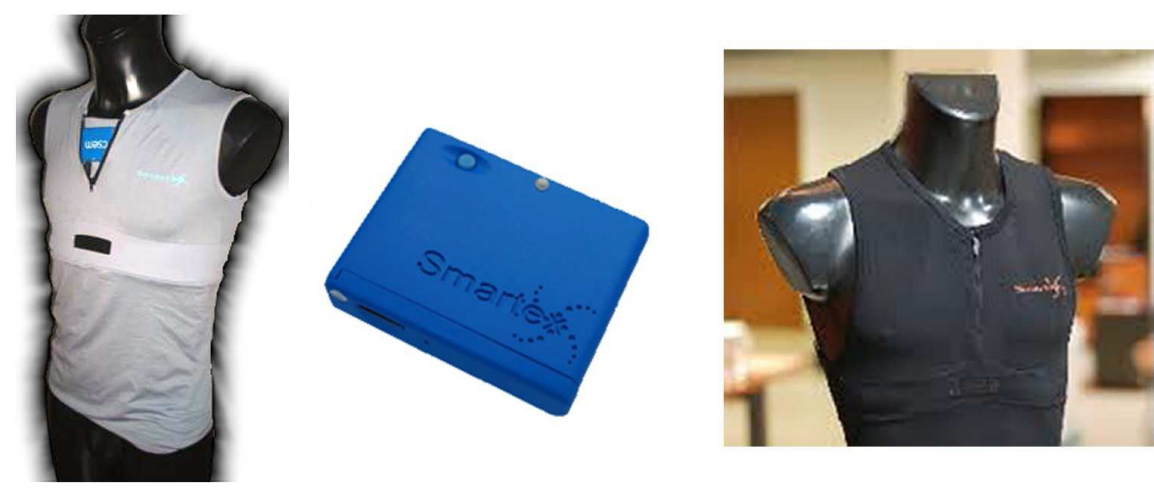

Figure 3. Sensorized t-shirt for acquiring ECG and respiration signals with the spatial coordinates of the subject (photo courtesy of Smartex).

Table 2. Raw and processed signals available from the Smartex t-shirt.

\begin{tabular}{|c|c|c|c|c|}
\hline \multicolumn{2}{|c|}{ Raw signals } & \multicolumn{3}{|c|}{ Processed signals } \\
\hline Name & $\begin{array}{l}\text { Rate } \\
(\mathrm{Hz})\end{array}$ & Name & $\begin{array}{l}\text { Rate }(\mathrm{Hz}) \\
\text { Period(s) }\end{array}$ & Unit \\
\hline ECG signal & 250 & ECG Quality & $5 \mathrm{~s}$ & 0-255 Quality index \\
\hline $\operatorname{Acc} X$ & 25 & Heart Rate & $5 \mathrm{~s}$ & Beats/min \\
\hline Acc Y & 25 & R-R Intervals & $\sim 1 \mathrm{~s}$ & \\
\hline Acc Z & 25 & ECG Enhanced Signal & $250 \mathrm{~Hz}$ & \\
\hline \multirow{10}{*}{$\begin{array}{c}\text { Respiration } \\
\text { Signal }\end{array}$} & \multirow{10}{*}{25} & Activity: Energy & $5 \mathrm{~s}$ & \\
\hline & & Activity: Type & $5 \mathrm{~s}$ & $\begin{array}{l}\text { Other } \\
\text { Lying } \\
\text { Standing/sitting } \\
\text { Walking } \\
\text { Intense physical activity }\end{array}$ \\
\hline & & Breath Rate & $15 \mathrm{~s}$ & \\
\hline & & Breath Amplitude & $5 \mathrm{~s}$ & \\
\hline & & Heart Rate Variability & $60 \mathrm{~s}$ & \\
\hline & & Respiration Signal & $5 \mathrm{~s}$ & 0-255 Quality index \\
\hline & & Activity: $1 /$ Pace & $1 \mathrm{~s}$ & Step period in $\mathrm{ms}$ \\
\hline & & Activity: Pace & $5 \mathrm{~s}$ & Steps/min \\
\hline & & ECG: Lead On/Off & $5 \mathrm{~s}$ & \\
\hline & & ECG: Resp-R & $\sim 1 \mathrm{~s}$ & \\
\hline
\end{tabular}

A software module called Physio Roblet connects the Smartex t-shirt to the HIPOP framework. The Physio Roblet manages the Bluetooth connection with the Smartex t-shirt acquisition unit, allowing experimenters to configure and test the connection before starting the experiment. All signals are transmitted by the electronic acquisition unit of the t-shirt as Bluetooth packets. To optimize the life of the battery, a transmission packet is sent when the electronic acquisition unit collects enough data to completely fill the packet. Consequently, data packets are received at a variable transmission rate. 
The Physio Roblet also includes real-time preprocessing algorithms that extract various timeand frequency-domain parameters. In particular, the ECG signal is filtered to remove typical ECG artifacts and is used to calculate R-R intervals (the distance between R peaks, also known as the tachogram) and HRV (heart rate variability). Since motion represents the most common source of artifacts in ECG, the accelerometer signals are used by dedicated algorithms to reject R-R values if strong movements are detected.

The Physio Roblet transmits the flow of data to the Bodymap for real-time visualization of the subject's psychophysiological state. Once the "record" message is received, the Physio Roblet stores the data locally as XML files. The Physio Roblet also accepts the "insert key point" message that triggers the writing of a time reference in the XML file.

\subsubsection{Multi-functional acquisition glove}

Our laboratory developed a multi-functional wearable glove for acquiring the electrodermal response (EDR), hand gestures, and postures (Valenza, Lanatà, Scilingo, \& De Rossi, 2010) along with an algorithm to infer the pleasure and arousal level of a subject involved in mental tasks and in manipulation and grasping (Valenza, Lanatà, \& Scilingo, 2011). The glove consists of two integrated textile electrodes in the index and middle fingers and an inertial multi-axial accelerometer in the upper arm/wrist (Fig. 4). The glove is worn on the non-dominant hand of the subject. Data are acquired through an electronic unit placed on the wrist, and the unit is equipped with a $\mathrm{ZigBee}{ }^{\mathrm{TM}}$ wireless communication system. The $\mathrm{ZigBee}^{\mathrm{TM}}$ signal is received by a dedicated dongle installed on a PC.

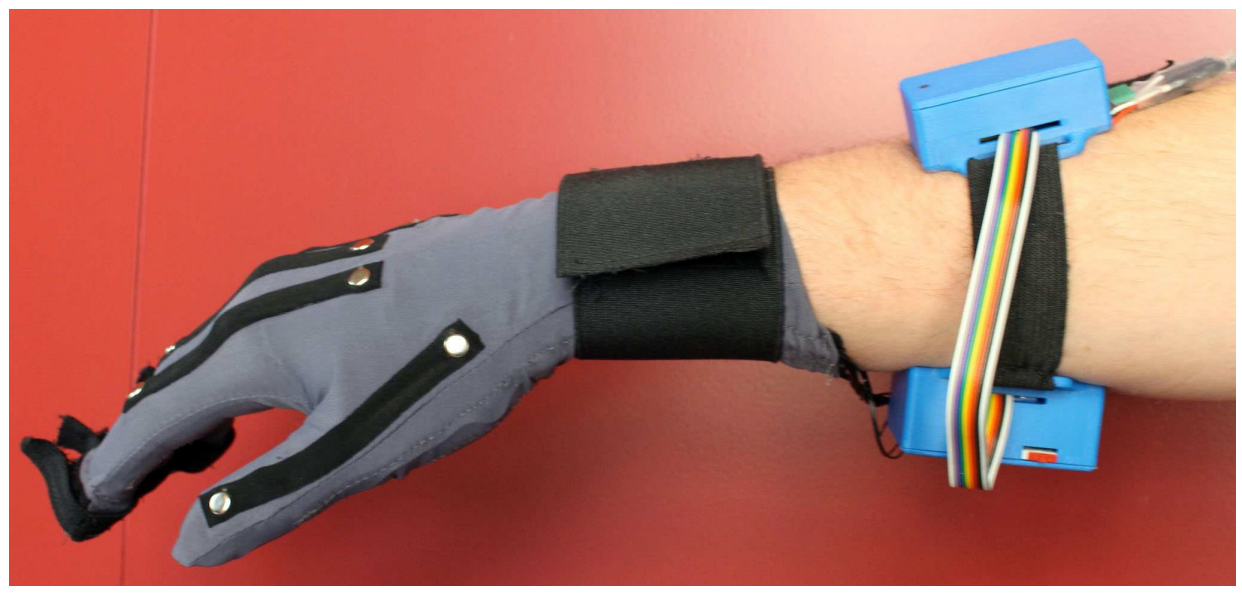

Figure 4. The multi-functional acquisition glove, which includes electrode sensors for EDR and sensors for reconstructing hand gestures and postures.

In the present embodiment and case study, a simpler and less unobtrusive version of the glove has been utilized that only measures, records and transmits the EDR.

Electrodermal activity (EDA) is a physiological signal that depends to a great degree on the local distribution of the eccrine sweat glands. Eccrine gland activity is controlled by the sympathetic nervous systems (SNS); therefore, EDA is considered a strong indicator of changes in the SNS in relation to emotions, feelings, cognition, and attention (Fowles, 1980; Rani, Sarkar, Smith, \& Kirby, 2004). The skin conductance signal has two components: (1) the tonic component that represents the baseline level and depends on the subject's physiological state and autonomic regulation, and (2) the phasic component that is related to the perception of external stimuli (Benedek \& Kaernbach, 2010). 
The software module called EDR Roblet manages the communication between the EDR acquisition system and the Bodymap, allowing experimenters to configure the system and visualize the acquired data in real-time. The system also includes a calibration routine used to adjust the electronic acquisition parameters to the subject's skin properties before starting the experiment. Once the EDR system is connected, it continuously sends the Bodymap acquired data for monitoring the subject's state. If the EDR Roblet receives the "record" message from the Bodymap, it records the acquired data in an XML file. If the EDR Roblet receives the "insert key point" message from the Bodymap, it records the time reference in an XML file.

\subsubsection{Eye tracking system}

Eye gaze and fixation time are known as fundamental cues that can be used to identify oculomotor characteristics and abnormalities in studies concerning behavioral and mental states. The system used in HIPOP is HATCAM, a wearable, highly comfortable, and minimally obtrusive eye tracking system designed and developed in our laboratory (Fig. 5).

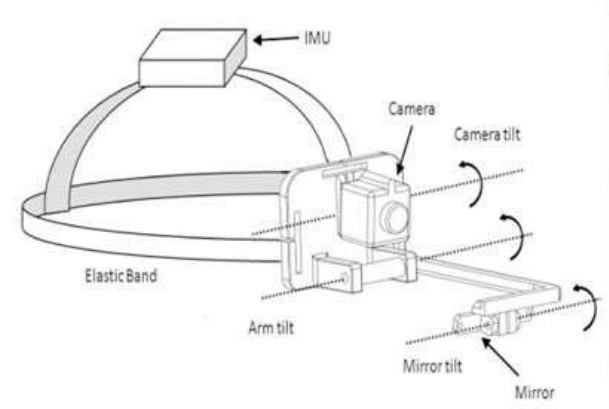

a

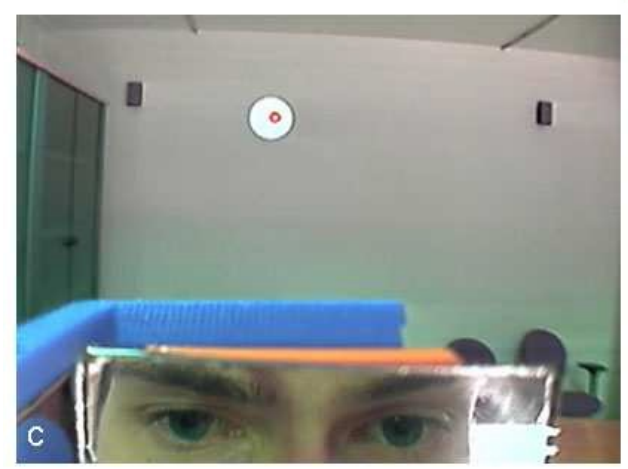

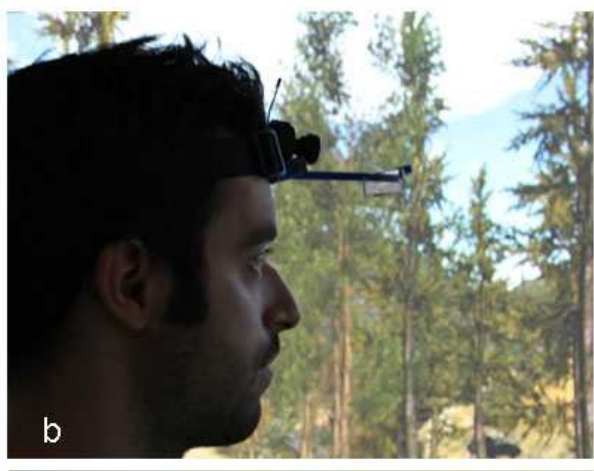

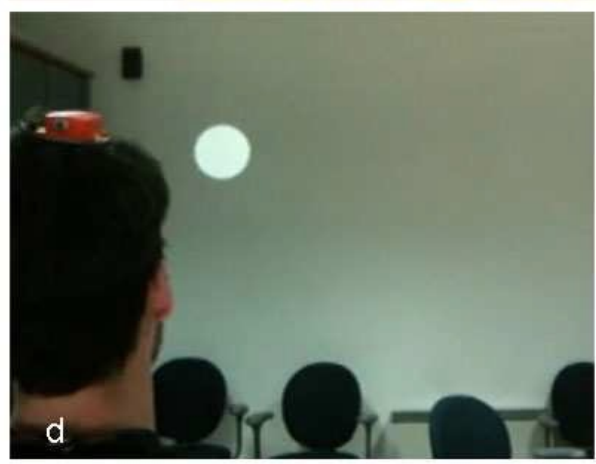

Figure 5. The HATCAM eye tracker. a) Schematic drawing b) Test in a virtual reality cave c) Gaze image d) Detail of the inertial platform.

The HATCAM consists of a child-sized cap or band equipped with a high-speed camera and an inertial platform. A small rectangular mirror with adjustable tilt is fixed on the brim and reflects eye movements back to the camera. The camera captures the scene in front of the subject and the pupils' image to track the eye gaze direction and other gaze-related parameters, such as the fixation time. A three-axis inertial platform is used to compensate for the vestibulo-ocular reflex and head movements (Armato, Lanatà, \& Scilingo, 2011). 
Preliminary testing of HATCAM as a tool for cognitive and affective analysis demonstrated the suitability of the system for use as a tool for affective and pupillometry studies (Lanatà, Armato, Valenza, \& Scilingo, 2011; Lanatà, Valenza, \& Scilingo, 2012).

A software module called HATCAM Roblet connects the HATCAM system to the Bodymap. Video recording is transmitted to a PC equipped with a USB video grabber through wireless connection. Data from the inertial platform are transmitted to the PC using a wireless ZigBee ${ }^{\mathrm{TM}}$ connection.

Once the HATCAM Roblet is started, it shows a preview of the video to allow experimenters to adjust the mirror position and perform the calibration routine. If the HATCAM is correctly calibrated, it shows the subject's field of view tracking the gaze point. If the HATCAM Roblet receives the "record" message from the Bodymap, it records the video and the gaze coordinates in an XML file. If the HATCAM Roblet receives the "insert key point" message from the Bodymap, it records the time reference in an XML file.

\subsubsection{Open-source module}

As previously mentioned, HIPOP is developed to be an open-source platform that allows experimenters to easily integrate both commercial and open-source acquisition devices or robot control systems.

A generic software module called OpenSource Roblet has been developed to connect hardware devices based on open-source systems, such as Arduino, RaspBerry Pi, or similar products with the Bodymap. Any kind of open-source hardware and software product that communicates through serial port can be integrated in HIPOP using its serial communication protocol. Data sent from the device to the computer serial port are acquired by the OpenSource Roblet and parsed according to the device communication protocol described in the OpenSource Roblet configuration file.

The OpenSource Roblet includes a basic GUI used by experimenters to configure the device and a set of charts to plot the acquired data. Experimenters can choose which data to plot on the charts by indicating the related channels in the OpenSource Roblet configuration file. The GUI can be easily extended, adding more charts allowing experimenters to visualize parallel data streams of different channels.

In HIPOP, the OpenSource Roblet has been instanced for acquiring data coming from an Arduino $\mathrm{UNO}^{1}$ board equipped with the e-Health platform produced by Cooking-Hacks ${ }^{2}$. The eHealth platform includes the acquisition of nine different biosensors: heart pulse and oxygen in blood saturation (SPO2), airflow (breathing), body temperature, electrocardiogram (ECG), glucose level, galvanic skin response (GSR), blood pressure and patient position. The e-Health sensor platform is provided with a high-level feature extraction and signal preprocessing API to easily extract signals directly on the Arduino board and stream data to the serial port.

The instance of OpenSource Roblet used in HIPOP only included the ECG and GSR sensors (Fig. 6). The communication protocol is a comma separated values (CSV) file that includes sample time, ECG and GSR values streamed at the appropriate serial rate (i.e., $115200 \mathrm{bps}$ ).

The OpenSource Roblet stores the selected channel data locally as XML files and transmits the flow of data to the Bodymap for real-time visualization on the Bodymap viewer.

\subsubsection{Bodymap viewer}

The Bodymap viewer is a graphical user interface (GUI) that shows all the data acquired by the HIPOP platform and shows the state of all the Roblets (Fig. 7). The Bodymap viewer also allows

\footnotetext{
${ }^{1}$ http://arduino.cc/

${ }^{2}$ http://www.cookinghacks.com/
} 
experimenters to set up the HIPOP experiment by selecting the type and number of Roblets to be activated and the computers on which they have to run.

The Bodymap viewer is used by experimenters to monitor the human-robot interaction in realtime, manage the recording activities, and annotate key points that are fundamental for the subsequent evaluation of the HRI experiment.

\subsubsection{HIPOP data management tool}

At the end of each experiment, each active Roblet receives a "stop" message to close and save its XML data files. Experimenters can collect all XML files from the various PCs that run the Roblets and merge them into a single XML data file using the HIPOP data management tool. This tool also allows experimenters to export each data channel as a separate CVS file that includes the desired channel together with the synchronized timestamp received by the Bodymap.

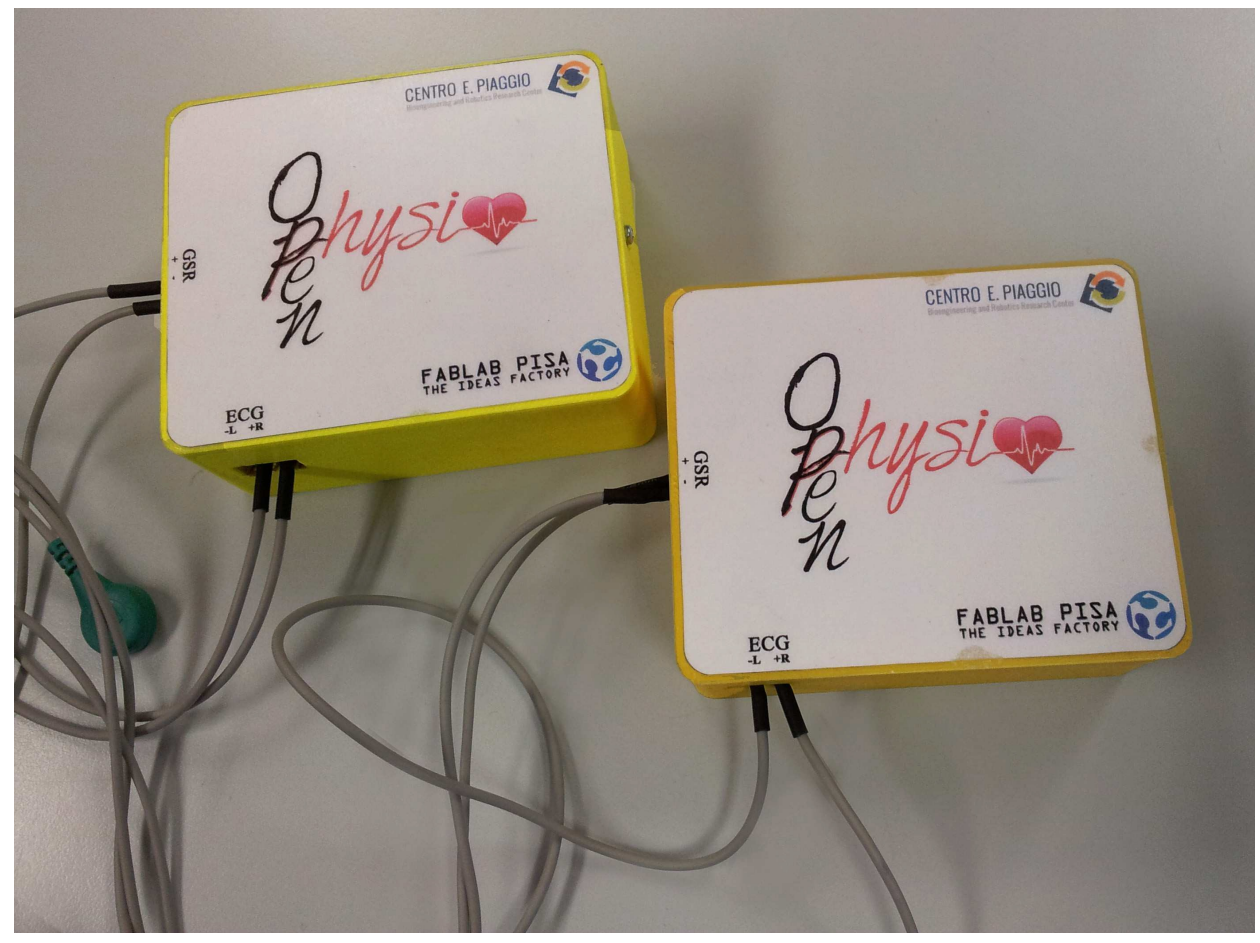

Figure 6. The Open Physio device built with an Arduino UNO board equipped with the eHealth platform, which acquires ECG and GSR signals.

A CSV file can be easily imported into many software analysis tools such as Kubios, LedaLab, and Matlab ${ }^{\mathrm{TM}}$ for extracting various high-level parameters and features that are fundamental for the subject's psychophysiological and behavioral state analysis.

\section{Technical Performance Evaluation}

The technical and functional capabilities of the HIPOP system were validated in terms of performance, error handling, and data management. The following fault conditions were reproduced to demonstrate the ability of HIPOP to auto-recover from failures: 
Hardware failures and Roblets crashes were simulated by disconnecting the peripheral power supply or killing a Roblet process. In both cases, the other Roblets continued to acquire, store, and forward data to the Bodymap without being affected by the failure of that Roblet. The Bodymap, which monitors the status of all Roblets, displayed a failure message indicating which Roblet crashed and updated the state of the Roblet on the Bodymap viewer. Once a peripheral power supply was restored or the process was restarted, a new instance of the Roblet was reconnected to the Bodymap and the data storing and streaming activities were restored.

Bodymap failures were simulated by killing the Bodymap process. In this case, all the Roblets continued to acquire data without being affected by the failure. Once the Bodymap was restarted, the data visualization and management was re-established.

Data synchronization among the Roblets running on different computers was verified by connecting various acquisition modules (the ECG, EDR, and Video/Audio Roblets) to a common signal source and analyzing the stored data alignment.

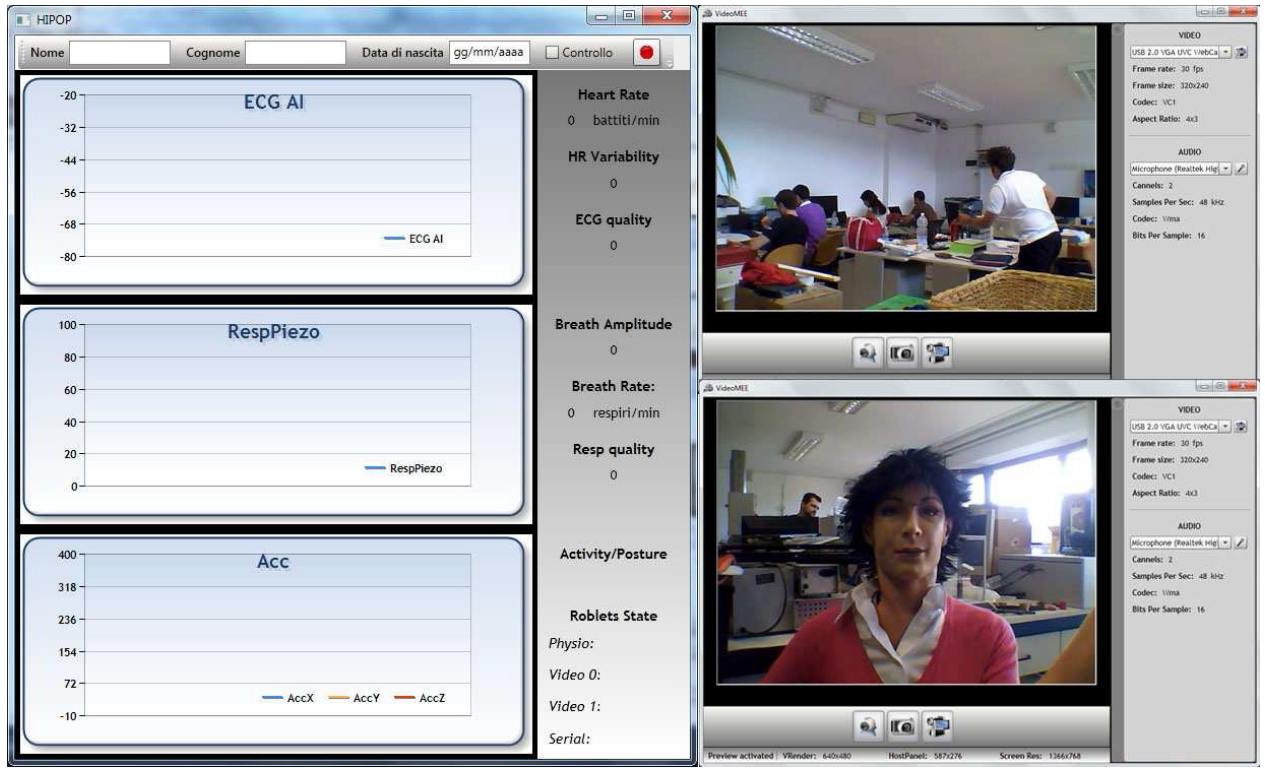

Figure 7. The Bodymap viewer shows the ECG, respiration, and accelerometer activity on the left and in the two videos on the right.

The equipment for the HIPOP synchronicity test was composed of three computers running four Roblets and the Bodymap, according to the following setup:

Computer 1: Bodymap and Physio Roblet

Computer 2: Video Roblet 1

Computer 3: Video Roblet 2 and EDR Roblet

A signal generator was connected to the t-shirt electrodes, the EDR finger electrodes, and the audio input channels of two computers, as described in Fig. 8.

The generated signals were sinusoidal waves at $20 \mathrm{~Hz}$ and $40 \mathrm{~Hz}$. Each signal was started and stopped every 30 seconds, 3 times each. The signal peaks were used as markers for signal misalignment evaluation. 


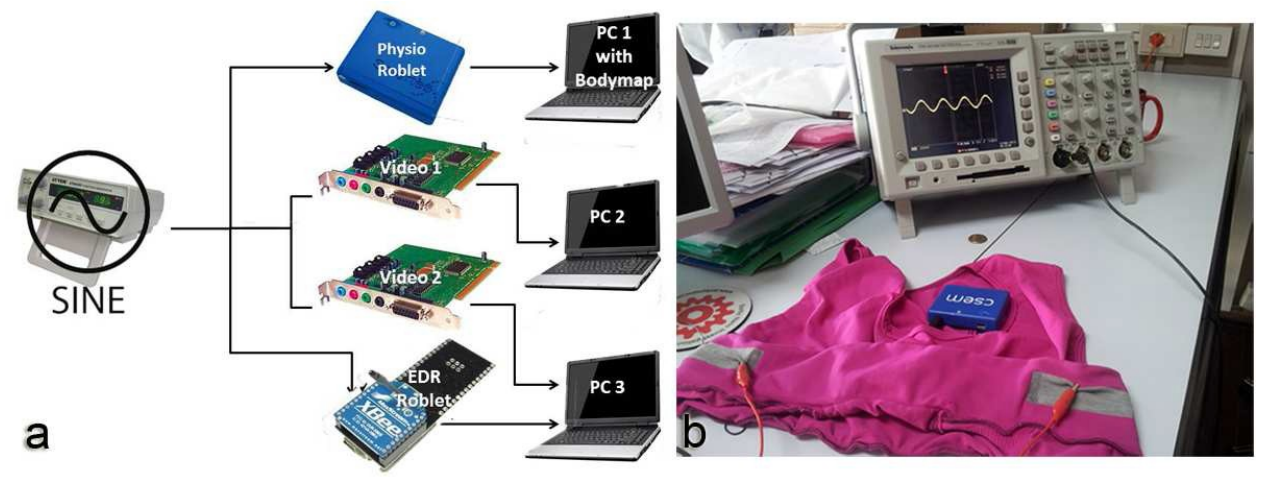

Figure 8. a) Scheme of hardware and software organization for the data synchronization test, b) the t-shirt with electrodes connected to the signal generator.

The EDR signal was acquired at $100 \mathrm{~Hz}$ and used as a reference for measuring misalignment. The ECG signal was acquired at $250 \mathrm{~Hz}$. The Video Roblet recorded at the rate of 25 FPS (frames per second) with a resolution of $720 \times 576$ pixels and the audio at $44.100 \mathrm{KHz}$ with a resolution of 16 bits. The tests were performed five times. All recorded data were imported into Matlab ${ }^{\text {тм }}$ to quantify the audio and EDR signal misalignment from the ECG signal. Time differences were calculated by measuring the distance from the last measured sine peak before the sine wave stopped (measurement points are indicated as black squares in Fig. 9).

The maximum misalignment was identified in the audio signal acquired using Video Roblet 1. The audio signal was recorded with a maximum delay of $57.4 \mathrm{~ms}$, due to the high computational workload required by the video streaming conversion and recording processes. This delay is acceptable if we consider the nature of the video signal, which is acquired at 25 FPS with a period of $40 \mathrm{~ms}$. Furthermore, video and audio signals are used as input for behavioral and psychological interpretation, which does not require great sharpness or precision. The misalignment also does not affect the audio processing because the signal integrity is guaranteed by the high frequency and resolution acquisition rate. The audio misalignment that was detected was lower than the typical affective audio features dynamic.

The ECG and EDR electronic units include low-pass filter stages with cutoffs at $50 \mathrm{~Hz}$ and 30 $\mathrm{Hz}$ respectively, and maximum acquirable periods of $20 \mathrm{~ms}$ and $33 \mathrm{~ms}$ respectively. The ECG signal had a maximum misalignment of $2.6 \mathrm{~ms}$ from the EDR, which is lower than the fastest acquirable signal period (Fig. 9).

\section{Case Study: FACET}

HIPOP has been used as an acquisition platform in the FACET (FACE Therapy) project. FACET is a social robot-based therapy for children with autism spectrum disorders (ASDs) and Asperger syndrome (Mazzei et al., 2010).

Theory, methods, and results of the FACET project are explained in detail in work by Mazzei, Greco, et al., (2012). In this section, we briefly presented and discussed the use of HIPOP in the FACET project as validation of its suitability when used as a tool in affective and social HRI studies.

As Fig. 10 shows, the HIPOP configuration used for the FACET project included four computers running the following software modules:

Computer 1: Bodymap

Computer 2: Video Roblet 1 and Physio Roblet

Computer 3: FACE Roblet

Computer 4: Video Roblet 2 and EDR Roblet 


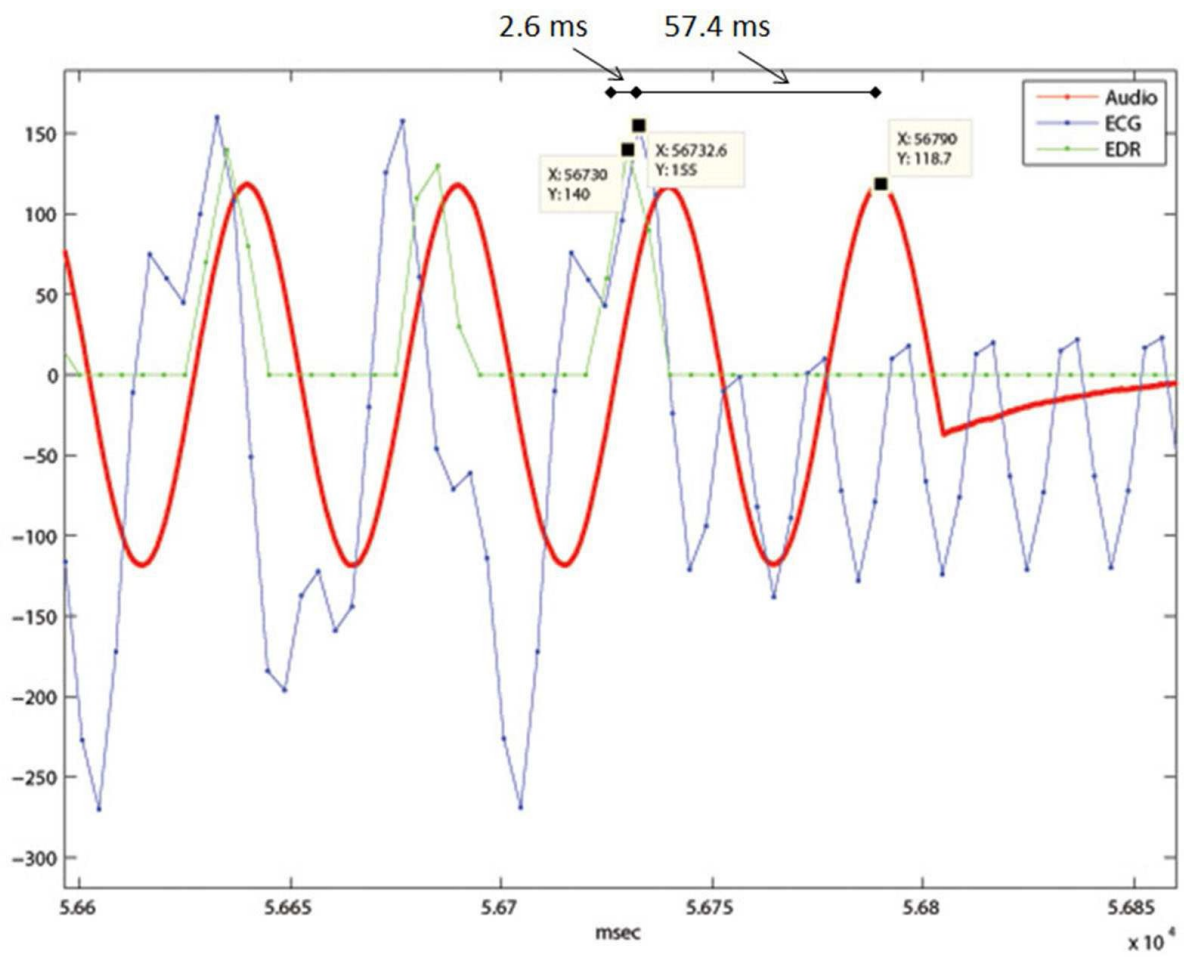

Figure 9. Signal alignment analysis. The EDR signal (green) was used as a reference to measure the time misalignment (ms) with the ECG signal (blue) and the audio (red) signal acquired by the Video Roblet 1.

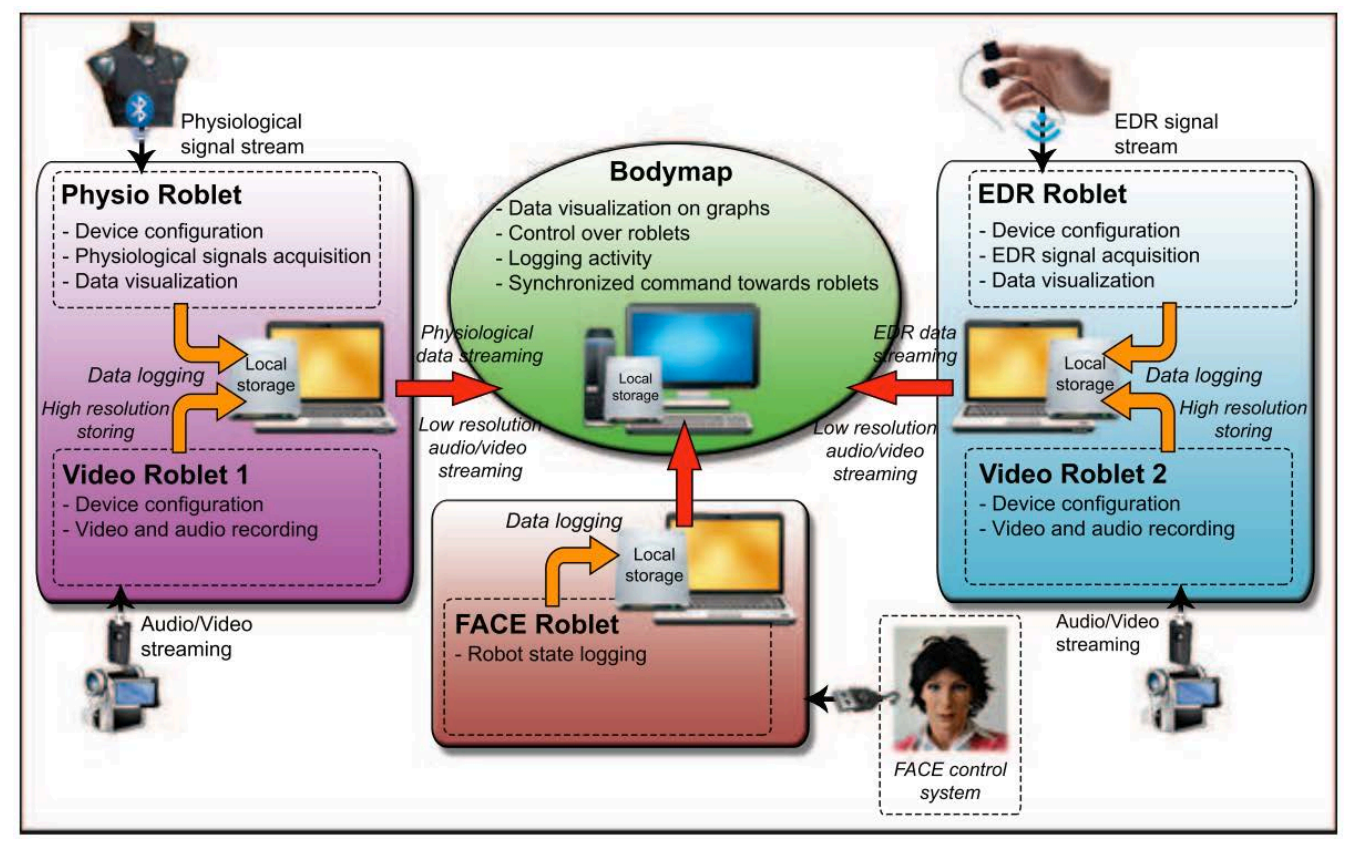

Figure 10. HIPOP configuration of the FACET project. 
The FACET project was based on the hypothesis that using a robot endowed with the ability to adapt and change its behavior or expressiveness according to a therapeutic protocol could train subjects with ASDs to gradually improve their social skills. The long-term goals were to improve the social and non-verbal communication skills of adolescents and young adults with ASDs (Mazzei et al., 2010, 2011; Mazzei, Greco, et al., 2012).

Indeed, subjects with ASDs or Asperger syndrome have difficulty understanding emotions and recognizing facial expressions, which are key elements in social interactions, because they provide guidance for identifying the intentions of others and managing interpersonal relationships and common social situations (Frith, 2003). Recent studies show that individuals with ASDs perceive robots as artificial playmates and not as machines (Scholtz, 2003). Based on this observation, researchers have considered the possibility of using robotic technology in rehabilitation therapies to improve focused attention, teach social behaviors, and increase the attention spans of children with ASDs (Breazeal \& Scassellati, 2000; Dautenhahn et al., 2009; Kozima, Nakagawa, \& Yano, 2005).

\subsection{Protocols and Methods}

The psychologists involved in the FACET project designed a protocol aimed at investigating the social and emotion recognition capabilities of subjects with ASDs during the interaction with the FACE android. The protocol was used to test whether the FACET platform was able to involve the subjects with ASDs in the interaction with the android without any discomfort. The FACET protocol was designed to acquire signals, video and annotations to be used by psychologists for inferring the subject's underlined state in terms of acceptance of the treatment and of understanding of the affective content of the designed interaction scenario.

The protocol was organized as a 25-minute-long session divided into 6 main phases:

1. Baseline acquisition: Physiological signals are acquired for 2-5 minutes in the absence of the FACE and social stimuli.

2. Familiarization: The therapist introduces the FACE and the therapeutic environment to the subject.

3. Labeling and imitation of the expressions of the FACE: The therapist invites the subject to label, describe, and imitate facial expressions performed by the FACE.

4. Labeling and imitation of the therapist's expressions: The therapist asks the subject to label, describe, and imitate the therapist's facial expressions.

5. Gaze cues processing: The gaze direction of the FACE is moved from the subject to a distant target to trigger the natural attention shift named "shared attention." The subject's reaction is evaluated in terms of attention shift and time required.

6. Spontaneous conversation and free play: The therapist invites the subject to play, touch, or question the FACE. This phase is intended to evaluate the subject's ability to engage in spontaneous conversations or play with the FACE or the psychologist.

The psychologists created an ad-hoc evaluation grid for assessing each task. For each therapeutic session, the grid reported the list of facial expressions performed by the FACE and by the psychologist, along with scores (i.e., 1 for a correct answer, 0 for a wrong answer) and additional notes about the subject's labeling and imitation activities.

The behavioral evaluation was conducted by comparing the analysis of all the videos recorded through the Video Roblets with the state of the FACE logged by the FACE Roblet and the psychologists' hand-written notes. The videos were also used to identify the beginning and end times of each protocol phase that were used as input for the physiological signals processing algorithms.

6.2 Data Analysis, Usability Evaluation, and Results

The FACET study was conducted by psychologists without technical and engineering backgrounds. The protocol involved children with ASDs of whom, in certain cases, did not easily 
accept acquisition devices and evaluation protocols. This can be considered as a first result of the HIPOP case study that demonstrates that the platform is highly usable, allowing experimenters to easily control a very complex and heterogeneous setup for a synchronized acquisition of both behavioral and physiological data.

Regarding to the HIPOP multimodal data analysis approach in the FACET project, video/audio files and annotations were manually analyzed by psychologists, while R-R, HRV, and GSR signals were processed by tools such as Kubios, LedaLab and Matlab ${ }^{\mathrm{TM}}$ to extract various time- and frequency-domain features (Valenza et al., 2011).

In particular, the following $\mathrm{R}-\mathrm{R}$ and $\mathrm{HRV}$ features were analyzed:

- Mean and standard deviation of R-R and HR

- HRV very low-frequency (VLF) power $(0-0.04 \mathrm{~Hz})$

- HRV low-frequency (LF) power $(0.04-0.15 \mathrm{~Hz})$

- HRV high-frequency (HF) power $(0.15-0.4 \mathrm{~Hz})$

- HRV LF/HF ratio $\left(\mathrm{ms}^{2} / \mathrm{ms}^{2}\right)$

ECG-derived parameters were analyzed as indicators of the sympathetic and parasympathetic nervous systems (SNS and PSNS) activities. In particular, HRV features are known to be correlated with the arousal component of the subject's emotional state. The LF/HF ratio that may be considered an indicator of the balance between the SNS and the PSNS (Malliani, Pagani, Lombard, \& Cerutti, 1991) can be extracted through a frequency-domain analysis.

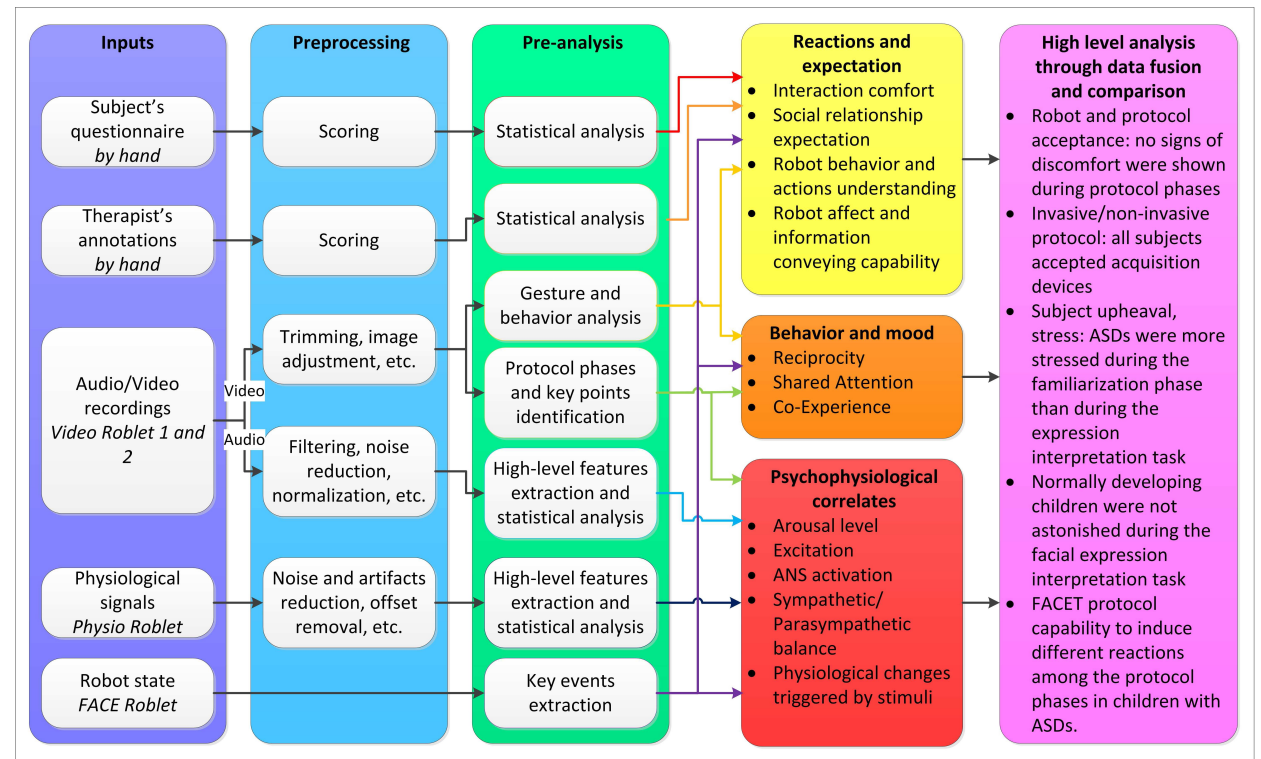

Figure 11. Data flow of the HIPOP multimodal analysis approach and framework used in the FACET project.

Fig. 11 shows an instance of the HIPOP multimodal analysis approach tailored for the FACET project. According to psychologists' reports and annotations (Mazzei et al., 2010, 2011), no children showed signs of discomfort during the FACET protocols, demonstrating the acceptability and unobtrusiveness of HIPOP. Analyzing the videos of each session and the state of the robot, psychologists were able to study the subjects' behaviors and attitudes toward establishing social relationships with the android and with the psychologist.

Video analysis and therapists' annotations revealed that some children with ASDs did not admit to being unable to understand the robot's expressions; however, analysis of LF/HF indicates that children with ASDs were more stressed during the familiarization phase than during the expression-interpretation task. The LF/HF ratio values showed that the stress condition was 
resolved during the familiarization phase. When children with ASDs were questioned about the FACE expressions, they were already confident with the scenario and not stressed by the expression-interpretation task.

In contrast, typically developing children were not astonished during the first interaction with the robot, most likely due to the similarity of the FACE to video games and movie characters. Instead, they faced the facial expression-interpretation task as a challenge, focusing on the analysis of the FACE expressions, which was confirmed by increased sympathetic activity (Hanson et al., 2012; Mazzei et al., 2010, 2011).

The high interest of children with ASDs in interacting with the FACE was also highlighted by the behavioral analysis results. During the shared attention phase, all children with ASDs followed the FACE, whereas only 60 percent of the control subjects did the same. During the conversation and free play phase, 55 percent of the children with ASDs established a spontaneous conversation with the FACE, whereas only 30 percent of the control subjects did the same.

High-level features extracted from ECG and GSR signal and processed according to protocol phases division were also used to perform a statistical analysis aimed at identifying inter-subject, inter-phases, and intra-subject correlations and differences (Mazzei, Greco, et al., 2012). Results showed that the two groups of subjects (children with ASDs and typically developing children) were statistically discernible according to the Kruskal-Wallis test (Kruskal \& Wallis, 1952). The Mann-Whitney test (Mann \& Whitney, 1947), on the features extracted from EDR, discerned the familiarization phase from the FACE expressions phase in children with ASDs. These results demonstrated the ability of the FACET protocol to induce different reactions among the protocol phases in children with ASDs. The Mann-Whitney test did not reveal any statistically significant differences among the protocol phases for the control group, which indicates that the protocol was well-designed to detect autistic behavioral differences.

The classification of these high-level features demonstrates how through the integrated multimodal analysis of HIPOP, it is possible to analyze both the subjects' reactions and underlying state together with group differences.

\section{Conclusions and Discussions}

HIPOP is a multimodal acquisition platform for pervasive HRI studies that is easy to use and configure. Thanks to its modular architecture and data fusion approach, the platform overcomes the usual data integration problems that commonly affect HRI setup. The focus on the open-source design can help to reduce the costs of HRI experiments. The integration of the open-source module for data acquisition through serial ports allows experimenters to use sensors, protocols, and systems of different brands as they integrate custom-developed or open-source prototypes.

HIPOP was shown to be flexible in configuring a custom setup and reliable in acquiring a large set of data. Tests on fault management demonstrated the ability of the platform to not propagate software or hardware faults to the other modules and to automatically recover from errors and failures. Tests on data synchronicity demonstrated that acquired signal misalignments were always lower than the fastest acquirable period in the case of physiological signals. Video acquisition showed the higher signal misalignment due to the high computational workload of the video acquisition process. This misalignment can be easily reduced using a computer with better performances and equipped with a dedicated video card. However, the measured video misalignment of 1.5 frames does not affect the behavioral analysis process.

HIPOP was used extensively in the context of the FACET project, and results demonstrated its stability in terms of technical performances along with its ease of usability when handled by psychologists without technical skills. The results of the FACET project also demonstrated the ability of the platform to acquire a large amount of data from different modules, thereby guaranteeing synchronicity. The multimodal approach facilitated by HIPOP allowed psychologists to investigate how children with autism interact with affective robots. Preliminary results demonstrated that through the integration of affective and behavioral studies, it was possible to 
infer how subjects with autism, in comparison with typically developing subjects, approached the FACE. Data acquired using HIPOP were also used to conduct a preliminary statistical and classification analysis of physiological signals with which it was also possible to partially discern subjects with autism from typically developing subjects.

These results indicate that HIPOP could become an innovative tool for HRI in social and affective studies, overcoming the usual challenges often associated with these experimental setups.

\section{Acknowledgements}

This work was partially funded by the European Commission under the 7th Framework Program project CEEDS, Project number: 258749, Call identifier: FP7-ICT-2009-5.

The authors would like to thank Professor F. Muratori, Dr. R. Igliozzi, and Dr. A. Mancini of the IRCCS Stella Maris-Scientific Institute of Developmental Neurology, Psychiatry, and Educational Psychology in Calambrone, Pisa (Italy).

\section{References}

Andreassi, J. L. (2000). Psychophysiology: Human behavior and physiological response (4th ed.). Mahwah, NJ: Lawrence Erlbaum Associates, Inc.

Armato, A., Lanatà, A., \& Scilingo, P. E. (2011). Comparative study on photometric normalization algorithms for an innovative, robust and real-time eye gaze tracker. Journal of RealTime Image Processing, 8, 1-13.

Atrey, P. K., Hossain, M. A., El Saddik, A., \& Kankanhalli, M. S. (2010). Multimodal fusion for multimedia analysis: A survey. Multimedia Systems, 16(6), 345-379. doi:10.1007/s00530-0100182-0

Benedek, M., \& Kaernbach, C. (2010). Decomposition of skin conductance data by means of nonnegative deconvolution. Psychophysiology, 47(4), 647-658. doi:10.1111/j.1469-

8986.2009.00972.x

Bethel, C., Burke, J., Murphy, R., \& Salomon, K. (2007). Psychophysiological experimental design for use in. human-robot interaction studies. In Proceedings of the International Symposium on Collaborative Technologies and Systems (CTS), 99-105. Orlando, FL.

doi:10.1109/CTS.2007.4621744

Bethel, C., \& Murphy, R. (2010). Review of human studies methods in HRI and recommendations. International Journal of Social Robotics, 2, 347-359. doi:10.1007/s12369-010-0064-9

Bradley, M. M., \& Lang, P. J. (1994). Measuring emotion: The self-assessment manikin and the semantic differential. Journal of Behavior Therapy and Experimental Psychiatry, 25 (1), 49-59. doi:10.1016/0005-7916(94)90063-9

Breazeal, C., \& Scassellati, B. (2000). Infant-like social interactions between a robot and a human caregiver. Adaptive Behavior, 8(1), 49-74. doi:10.1177/105971230000800104

Cacioppo, J. T. (2007). Handbook of psychophysiology (3rd ed.), pp. 581-607. New York, NY: Cambridge University Press.

Cisternino, A., Colombo, D., Ennas, G., \& Picciaia, D. (2005). Robotics4.NET: Software body for controlling robots. IEE Proceedings, Software, 152(5), 215-222. doi:10.1049/ip-sen:20045030

Damasio, A. (1994). Descartes' error: Emotion, reason, and the human brain. New York, NY: Grosset/Putnam.

Damasio, A. (2005, April). Feeling our emotions. Scientific American. Retrieved from www.scientificamerican.com/article.cfm?id=feeling-our-emotions 
Dautenhahn, K., Nehaniv, C., Walters, M., Robins, B., Kose-Bagci, H., Mirza, N., \& Blow, M. (2009). KASPAR - A minimally expressive humanoid robot for human-robot interaction research. Applied Bionics and Biomechanics, 6, 369-397. doi:10.1080/11762320903123567

Dautenhahn, K., \& Werry, I. (2002). A quantitative technique for analysing robot-human interactions. In Proceedings of the IEEE/RSJ International Conference on Intelligent Robots and Systems, 2, 1132-1138. doi:10.1109/IRDS.2002.1043883

Fowles, D. C. (1980). The three arousal model: Implications of gray's two-factor learning theory for heart rate, electrodermal activity, and psychopathy. Psychophysiology, 17 (2), 87-104. doi:10.1111/j.1469-8986.1980.tb00117.x

Frith, U. (2003). Autism, explaining the enigma (2nd ed.). Oxford, UK: Blackwell.

Hanson, D., Mazzei, D., Garver, C., Ahluwalia, A., De Rossi, D., Stevenson, M., \& Reynolds, K. (2012). Realistic Human-like robots for treatment of ASD, social training, and research; Shown to appeal to youths with ASD, cause physiological arousal, and increase human-to-human social engagement. In Proceedings of the 5th ACM International Conference on Pervasive Technologies Related to Assistive Environments (PETRA). New York, NY.

Jaimes, A., \& Sebe, N. (2007). Multimodal human-computer interaction: A survey. Computer Vision and Image Understanding, 108 (1-2), 116-134. doi:10.1016/j.cviu.2006.10.019

Kanda, T., Ishiguro, H., Imai, M., \& Ono, T. (2003). Body movement analysis of human-robot interaction. In Proceedings of the 18th International Joint Conference on Artificial Intelligence (IJCA), 177-182. San Francisco, CA: Morgan Kaufmann Publishers Inc. Retrieved from http://dl.acm.org/citation.cfm?id=1630659.1630684

Kanda, T., Miyashita, T., Osada, T., Haikawa, Y., \& Ishiguro, H. (2008). Analysis of humanoid appearances in human-robot interaction. IEEE Transactions on Robotics, 24 (3), 725-735. doi:10.1109/TRO.2008.921566

Kidd, C., \& Breazeal, C. (2005). Human-robot interaction experiments: Lessons learned. In K. Dautenhahn \& R. de Boekhorst (Eds.), Robot Companions: Hard Problems and Open Challenges in Robot-Human Interaction Symposium at Social Intelligence and Interaction in Animals, Robots and Agents (AISB), 141-142. University of Hertfordshire, Hatfield, UK.

Kim, J., \& André, E. (2008). Emotion recognition based on physiological changes in music listening. IEEE Transactions on pattern analysis and machine intelligence, 30, 2067-2083. doi:10.1109/TPAMI.2008.26

Kim, K., Bang, S., \& Kim, S. (2004). Emotion recognition system using short-term monitoring of physiological signals. Medical and Biological Engineering and Computing, 42 (3), 419-427.

Kozima, H., Nakagawa, C., \& Yano, H. (2005). Using robots for the study of human social development. In Proceedings of the AAAI Spring Symposium on Developmental Robotics (DevRob), 111-114. Palo Alto: CA.

Kruskal, W., \& Wallis, W. (1952). Use of ranks in one-criterion variance analysis. Journal of the American Statistical Association, 583-621. doi:10.1080/01621459.1952.10483441

Lanatà, A., Armato, A., Valenza, G., \& Scilingo, E. P. (2011). Eye tracking and pupil size variation as response to affective stimuli: A preliminary study. In Proceedings of the 5th International Conference on Pervasive Computing Technologies for Healthcare (PervasiveHealth), 78-84. New York, NY.

Lanatà, A., Valenza, G., \& Scilingo, E. (2012). Eye gaze patterns in emotional pictures. Journal of Ambient Intelligence and Humanized Computing, 4(6), 705-715.

Ledoux, J. (1998). The emotional brain: The mysterious underpinnings of emotional life. Simon \& Schuster. 
Lazerri et al., Multimodal Acquisition Platform

Lisetti, C., \& Nasoz, F. (2002). MAUI: a multimodal affective user interface. In Proceedings of the 10th ACM International Conference on Multimedia, 161-170. New York, NY.

Lohse, M., Hanheide, M., Rohlfing, K. J., \& Sagerer, G. (2009). Systemic interaction analysis (SInA) in HRI. In Proceedings of the 4th ACM/IEEE International Conference on Human Robot Interaction, 93-100. New York, NY.

Malliani, A., Pagani, M., Lombardi, F., \& Cerutti, S. (1991). Cardiovascular neural regulation explored in the frequency domain. Circulation, 84, 482-492. doi:10.1161/01.CIR.84.2.482

Mann, H. B., \& Whitney, D. R. (1947). On a test of whether one of two random variables is stochastically larger than the other. Annals of Mathematical Statistics, 18, 50-60.

Mazzei, D., Billeci, L., Armato, A., Lazzeri, N., Cisternino, A., Pioggia, G., . . De Rossi, D. (2010). The FACE of autism. In Proceedings of the 19th IEEE International Symposium on Robot and Human Interactive Communication (RO-MAN), 791-796. New York, NY. doi:10.1109/ROMAN.2010.5598683

Mazzei, D., Greco, A., Lazzeri, N., Zaraki, A., Lanatà, A., Igliozzi, R., De Rossi, D. (2012). Robotic social therapy on children with autism: Preliminary evaluation through multi-parametric analysis. In Proceedings of the International Conference on Social Computing (SocialCom): Privacy, Security, Risk and Trust (PASSAT), 955-960. New York, NY. doi:10.1109/SocialComPASSAT.2012.99

Mazzei, D., Lazzeri, N., Billeci, L., Igliozzi, R., Mancini, A., Ahluwalia, A., Muratori, F., \& De Rossi, D. (2011). Development and evaluation of a social robot platform for therapy in autism. In Proceedings of the 33rd Annual International Conference of the IEEE Engineering in Medicine and Biology Society (EMBC) (pp. 4515-4518). New York, NY.

doi:10.1109/IEMBS.2011.6091119

Mazzei, D., Lazzeri, N., Hanson, D., \& De Rossi, D. (2012). HEFES: A hybrid engine for facial expressions synthesis to control human-like androids and avatars. In Proceedings of the 4th IEEE $R A S \&$ EMBS International Conference on Biomedical Robotics and Biomechatronics (BioRob), 195-200. New York, NY. doi:10.1109/BioRob.2012.6290687

Paradiso, R., Loriga, G., \& Taccini, N. (2005). A wearable health care system based on knitted integrated sensors. IEEE Transactions on Information Technology in Biomedicine, 9(3), 337-344. doi:10.1109/TITB.2005.854512

Rani, P., Sarkar, N., Smith, C. A., \& Kirby, L. D. (2004). Anxiety detecting robotic system towards implicit human-robot collaboration. Robotica, 22(1), 85-95.

doi:10.1017/S0263574703005319

Robinson, P., \& El Kaliouby, R. (2009). Computation of emotions in man and machines.

Philosophical Transactions of the Royal Society B: Biological Sciences, 364, 3441-3447. doi:10.1098/rstb.2009.0198

Scholtz, J. (2003). Theory and evaluation of human-robot interaction. In Proceedings of the 36th Hawaii International Conference on System Sciences (HICSS), 36, 125-134. New York, NY. doi:10.1109/HICSS.2003.1174284

Scilingo, E. P., Gemignani, A., Paradiso, R., Taccini, N., Ghelarducci, B., \& De Rossi, D. (2005). Performance evaluation of sensing fabrics for monitoring physiological and biomechanical variables. IEEE Transactions on Information Technology in Biomedicine, 9(3), 345-352. doi:10.1109/TITB.2005.854506

Steinfeld, A., Fong, T., Kaber, D., Lewis, M., Scholtz, J., Schultz, A., \& Goodrich, M. (2006). Common metrics for human-robot interaction. In Proceedings of the 1st ACM SIGCHI/SIGART Conference on Human-Robot Interaction, 33-40. New York, NY. doi:10.1145/1121241.1121249 
Stern, R., Ray, W., \& Quigley, K. (2001). Psychophysiological recording (2nd ed.). Oxford, UK: Oxford University Press, Inc.

Tiberio, L., Cesta, A., \& Olivetti Belardinelli, M. (2013). Psychophysiological methods to evaluate user's response in human robot interaction: A review and feasibility study. Robotics, 2(2), 92-121. doi:10.3390/robotics2020092

Valenza, G., Lanatà, A., \& Scilingo, E. P. (2011). The role of nonlinear dynamics in affective valence and arousal recognition. IEEE Transactions on Affective Computing, 99 (PrePrints). doi:10.1109/T-AFFC.2011.30

Valenza, G., Lanatà, A., Scilingo, E. P., \& De Rossi, D. (2010). Towards a smart glove: Arousal recognition based on textile electrodermal response. In Proceedings of the 32nd Annual International Conference of the IEEE Engineering in Medicine and Biology Society (EMBC), 3598-3601. New York, NY.

Wienke, J., Klotz, D., \& Wrede, S. (2012). A framework for the acquisition of multimodal humanrobot interaction data sets with a whole-system perspective. In Proceedings of the LREC Workshop on Multimodal Corpora for Machine Learning. Istanbul, Turkey.

Nicole Lazzeri, Research Center "E. Piaggio," University of Pisa, Italy. Email: n.lazzeri@centropiaggio.unipi.it; Daniele Mazzei, Research Center "E. Piaggio," University of Pisa, Italy. Email: mazzei@di.unipi.it; Danilo De Rossi, Research Center "E. Piaggio," University of Pisa, Italy. Email: d.derossi@centropiaggio.unipi.it 